\title{
CARACTERIZAÇÃO DOS PRODUTOS GERADORES DE RESÍDUOS QUÍMICOS PERIGOSOS: ESTUDO EM UM HOSPITAL PÚBLICO UNIVERSITÁRIO*
}

Taiza Florêncio Costa ${ }^{1}$, Vanda Elisa Andres Felli², Angela Maria Magosso Takayanaguii ${ }^{3}$, Leny Borghesan Albertini ${ }^{4}$, Patrícia Campos Pavan Baptista ${ }^{5}$

RESUMO: Estudo descritivo com o objetivo de identificar nas áreas de farmácia e almoxarifado de um Hospital Universitário os grupos de produtos caracterizados como geradores de Resíduos Químicos Perigosos segundo a classificação dos dispositivos legais. Para coleta de dados, realizada em 2008, foi utilizado um formulário para identificação destes produtos. A análise dos dados revelou a existência de 138 itens classificados em 12 Grupos de produtos que, uma vez utilizados ou vencidos, geram resíduos com características de periculosidade de acordo com a Agência Nacional de Vigilância Sanitária brasileira. O princípio ativo desses produtos apresentam riscos à saúde, como toxicidade aos diversos órgãos e sistemas do corpo humano, assim como potencial de inflamabilidade, corrosividade e reatividade de algumas substâncias. Essas características são de grande relevância, uma vez que os resíduos químicos poderão, em algum momento, causar impacto negativo para a saúde do trabalhador, saúde pública e meio ambiente. DESCRITORES: Resíduos químicos; Saúde ocupacional; Saúde pública; Saúde ambiental.

\section{CHARACTERIZATION OF PRODUCTS WHICH CREATE DANGEROUS CHEMICAL WASTE: A STUDY IN A PUBLIC UNIVERSITY HOSPITAL}

\begin{abstract}
This descriptive study aimed to identify, in the areas of Pharmacy and the stockroom of a Public University Hospital, the groups of products characterized as creating Dangerous Chemical Waste, according to the legal classification. A form for identifying these products was used for data collection, which took place in 2008. Analysis of the data revealed the existence of 138 items classified in 12 Groups of products which, once used or out of date, create waste with dangerous characteristics, according to the Brazilian National Health Surveillance Agency. These products' active ingredients present risks to health, such as toxicity to various organs or systems in the human body, as well as the potential for inflammability, corrosiveness and reactivity of some substances. These characteristics are highly relevant, as the chemical wastes can, at some point, impact negatively on the worker's health, on public health, or on the environment.

DESCRIPTORS: Chemical waste; Occupational health; Public health; Environmental health.

\section{CARACTERIZACIÓN DE LOS PRODUCTOS GENERADORES DE RESIDUOS QUÍMICOS PELIGROSOS: ESTUDIO EN UN HOSPITAL PÚBLICO UNIVERSITARIO}

RESUMEN: Estudio descriptivo cuyo objetivo fue identificar en las áreas de farmacia y depósito de un Hospital Universitario los grupos de productos caracterizados como generadores de Residuos Químicos Peligrosos de acuerdo a la clasificación de los dispositivos legales. Para recoger los datos, lo que se dio en 2008, fue utilizado un formulario que identificaba eses productos. El análisis de los datos reveló la existencia de 138 ítenes clasificados en 12 Grupos de productos que, una vez utilizados o vencidos, generan residuos con características de peligrosidad de acuerdo con la Agencia Nacional de Vigilancia Sanitaria brasileña. El principio activo de eses productos presenta riesgos a la salud, como si es tóxico a los diversos órganos y sistemas del cuerpo humano, así como potencial de inflamabilidad, corrosividad y reactividad de algunas substancias. Esas características son de grande relevancia, una vez que los residuos químicos podrán, en algun momento, causar impacto negativo a la salud del trabajador, salud pública y medio ambiente.

DESCRIPTORES: Residuos químicos; Salud ocupacional; Salud pública; Salud ambiental.

*Extraído da tese 'Gerenciamento de resíduos químicos perigosos manuseados pela enfermagem de um Hospital Universitário' apresentada à Escola de Enfermagem da Universidade de São Paulo-EE USP em 2009.

${ }^{1}$ Enfermeira. Doutora em Ciências. Pós-Doutoranda pelo Programa Nacional de Pós-Doutorado-PNPD CAPS da EE USP.

${ }^{2}$ Enfermeira. Doutora em Enfermagem. Professora do Departamento de Orientação Profissional e do Programa de Pós-Graduação em Enfermagem da EE USP.

${ }^{3}$ Enfermeira. Doutora em Enfermagem. Professora responsável pelo Laboratório de Saúde Ambiental da Escola de Enfermagem de Ribeirão Preto da USP.

${ }^{4}$ Química. Gerente responsável técnica do Laboratório de Tratamento de Resíduos Químicos da Território Ambiental.

${ }^{5}$ Enfermeira. Doutora em Enfermagem. Professora do Departamento de Orientação Profissional e do Programa de Pós-Graduação da EE USP.

Autor correspondente:

Recebido: $18 / 06 / 2012$

Taiza Florêncio Costa

Aprovado: 10/01/2013

Universidade de São Paulo

R.. Dr. Enéas de Carvalho Aguiar, 419 - 05403-000 -São Paulo-SP-Brasil

E-mail: taizaflorencio@usp.br 


\section{INTRODUÇÃO}

Na última década, com maior ênfase, vem se vivenciando mudanças climáticas intensas que apontam para diversas formas de destruição do planeta. Estes eventos, decorrentes do desenvolvimento social, podem ser minimizados pela ação humana consciente. No final da década de 1960 já havia uma tímida preocupação quanto à importância e cuidados especiais a serem dispensados aos resíduos dos serviços de saúde ${ }^{(1)}$.

Para caracterizar seus produtos geradores de resíduos químicos perigosos as instituições hospitalares devem lançar mão dos critérios estabelecidos pela Resolução 306 da Agência Nacional de Vigilância Sanitária (ANVISA), de 2004(2); da Companhia de Tecnologia de Saneamento Ambiental (CETESB) por meio da Norma Técnica P4.262/2007(3) assim como da Portaria 21 do Centro de Vigilância Sanitária (CVS) de 10/09/2008 ${ }^{(4)}$. Ainda, na caracterização dos produtos químicos geradores de resíduos químicos perigosos destaca-se a Norma da Associação Brasileira de Normas Técnicas (ABNT) intitulada Produtos Químicos - Informações sobre Segurança, Saúde e Meio Ambiente ${ }^{(5)}$. Embasando os dispositivos legais, a Norma Brasileira Regulamentadora (NBR) 10.004 estabelece que se pode considerar um resíduo químico hospitalar perigoso quando apresentar, pelo menos, uma das seguintes propriedades: inflamabilidade, corrosividade, reatividade, e toxicidade ${ }^{(6)}$. Esta Norma fundamenta a Resolução CONAMA $^{(7)}$ 358/2005 que dispõe sobre o tratamento e a disposição final dos resíduos de serviços de saúde e os classifica em Grupos A, B, C, D, E. Pertencem ao Grupo B os resíduos contendo substâncias químicas que podem apresentar risco à saúde pública ou ao meio ambiente, dependendo de suas características de periculosidade. Os resíduos químicos com características de periculosidade, quando não forem submetidos a processo de reutilização, recuperação ou reciclagem, devem ser submetidos a tratamento e disposição final específicos ${ }^{(3-4)}$.

Atualmente, observa-se a busca na harmonização entre o CONAMA e os demais órgãos como a ABNT e ANVISA. A Resolução ANVISA 306/04(2) estabelece que todo gerador de Resíduo de Serviços de Saúde(RSS) deverá elaborar um Plano de Gerenciamento de Resíduos de Serviços de Serviços de Saúde (PGRSS). Esse é o documento que aponta e descreve as ações relativas ao manejo dos resíduos sólidos, observadas suas características e riscos, no âmbito dos estabelecimentos, ainda contempla, os aspectos referentes à geração, segregação, acondicionamento, coleta, armazenamento, transporte, tratamento e disposição final, bem como as ações de proteção à saúde pública e ao meio ambiente. De forma ampla entende-se por manejo dos RSS a ação de gerenciar os resíduos em seus aspectos intra e extra estabelecimento, desde a geração até a disposição final $^{(2)}$. Segundo a ANVISA ${ }^{(8-9)}$ cabe ao gerador indicar a destinação e a forma de tratamento externo pretendidos para recuperação, descarte, incineração ou aterros industriais, uma vez que há grande interesse em se adotar novos procedimentos para eliminação e reaproveitamento das substâncias químicas, com repercussão direta na economia e na segurança química do local. É recomendado ainda que o gerador prepare um guia prático, buscando alternativas de neutralização, baseado na Ficha de Informações de Segurança de Produtos Químicos (FISPQ) ${ }^{(5)}$.

Nesse contexto destaca-se a Norma Regulamentadora $32^{(10)}$, que trata da segurança e saúde no trabalho em serviços de saúde.

Frente à periculosidade dos resíduos à saúde humana e ambiental e das lacunas de estudos quanto a caracterização dos produtos químicos geradores de resíduos perigosos hospitalares bem como da obrigatoriedade legal do estabelecimento gerador de RSS em gerenciar seus resíduos ${ }^{(2)}$, buscou-se no presente estudo identificar os produtos geradores de resíduos químicos perigosos (RQP) segundo critérios propostos pelos dispositivos legais estabelecidos pela política nacional de gestão de resíduos.

\section{MÉTODO}

Trata-se de uma pesquisa descritiva, uma vez que pretende demonstrar a existência de princípios ativos potencialmente geradores de resíduos químicos perigosos segundo a classificação do Grupo B da RDC 306/04 da ANVISA(2), Portaria CVS21 $/ 08^{(4)}$ e Norma CETESB P4.262/07(3). A instituição campo de estudo foi o Hospital Universitário da Universidade de São Paulo (HU USP).

Os dados foram coletados no período de abril a junho de 2008, utilizando-se o instrumento denominado 'Formulário para identificação nos estoques dos produtos químicos de uso médico-hospitalar' do almoxarifado e farmácia do HU USP.

Após aprovação do projeto pelo Comitê de Ética e Pesquisa do HU USP, protocolo de número 785/07 e contato com a direção do serviço de farmácia e almoxarifado, os dados foram coletados. Os produtos foram caracterizados, classificados e analisados segundo os Grupos dos produtos hormonais, antimicrobianos, antineoplásicos, imunomoduladores, imunossupressores, anti-retrovirais, efluentes de processadores, digitálicos; medicamentos da portaria MS 344/98, desinfetantes, esterilizantes e demais produtos perigosos que repre- 
sentem princípios ativos geradores de resíduos químicos perigosos à saúde humana e meio ambiente ${ }^{(3)}$.

\section{RESULTADOS}

Os dados permitiram identificar os Grupos de produtos com princípios ativos geradores de RQP, por meio dos critérios de classificação dos dispositivos legais de gerenciamento de RSS. Foram geradas três tabelas e um quadro os quais evidenciam tal caracterização.

$\mathrm{O}$ registro dos itens de estoque classificados no Grupo B da ANVISA podem ser visualizados na tabela 1 e na qual verifica-se o total de 134 itens dos estoques da farmácia e almoxarifado, organizados em 12 grupos classificatórios preconizados pela RDC306/04 da ANVISA $^{(2)}$ como Grupo B dos resíduos químicos.

Observa-se a predominância de itens nos grupos de produtos geradores de RQP entre os antimicrobianos, medicamentos da portaria MS 344/98 ${ }^{(8)}$ e demais produtos perigosos respectivamente, sendo que o grupo dos demais produtos perigosos inclui itens inflamáveis, corrosivos, reativos ou tóxicos que não se classificam em nenhum dos outros 11 grupos.

Tabela 1 - Frequência dos itens de estoque dos produtos químicos perigosos, segundo o Grupo B da RDC 306/04 da ANVISA. São Paulo, 2008

\begin{tabular}{lcc}
\hline Grupo & $\mathbf{n}$ & $\mathbf{\%}$ \\
\hline Antimicrobiano & 47 & 33,81 \\
Antineoplásico & 2 & 1,43 \\
Anti-retroviral & 5 & 3,59 \\
Desinfetantes & 2 & 1,43 \\
Digitálico & 1 & 0,71 \\
Efluentes de processadores & 2 & 1,43 \\
Esterilizantes & 2 & 1,43 \\
Hormonal & 6 & 4,31 \\
Imunomodulador & 1 & 0,71 \\
Imunossupressor & 2 & 1,43 \\
Medicamentos da portaria 344/98 & 43 & 31,65 \\
Demais produtos perigosos & 21 & 17,98 \\
Total & 134 & 100 \\
\hline
\end{tabular}

Verifica-se na tabela 2 o total de 13 itens dos estoques da farmácia e almoxarifado, organizados em 5 grupos classificatórios preconizados pela Portaria CVS $21 / 08^{(4)}$ como produtos geradores de RQP. Observa-se a predominância dos grupos hormonais e demais produtos perigosos, sendo que nesse último são incluídos somente os produtos medicamentosos geradores de resíduos perigosos e que não se classificam em nenhum outro grupo da Portaria CVS 21/08(4).
Tabela 2 - Frequência princípios ativos de produtos químicos perigosos, segundo os grupos da Portaria CVS 21/08. São Paulo, 2008

\begin{tabular}{|c|c|c|c|}
\hline Grupo & Princípio Ativo & $\mathbf{n}$ & $\%$ \\
\hline Antimicrobiano & Cloranfenicol & 1 & 30,76 \\
\hline Antineoplásico & Ganciclovir, Ciclofosfamida & 2 & 7,69 \\
\hline Anti-retroviral & Zidovudina & 1 & 15,38 \\
\hline Hormonal & $\begin{array}{l}\text { Ergometrina, ocitocina, pro- } \\
\text { gesterona, propiltiouracila }\end{array}$ & 4 & 7,69 \\
\hline $\begin{array}{l}\text { Demais } \\
\text { Produtos }\end{array}$ & $\begin{array}{l}\text { Colchicina, epinefrina, } \\
\text { nicotina, nitroglicerina, } \\
\text { varfarina }\end{array}$ & 5 & 38,46 \\
\hline Total & & 13 & 100 \\
\hline
\end{tabular}

Observa-se na tabela 3, o registro dos princípios ativos nos Grupos de produtos que conferem periculosidade a seus resíduos, segundo a classificação da Norma CETESB/ P4.262 ${ }^{(3)}$. Verifica-se na Tabela 3, o total de 19 princípios ativos identificados nos estoques, os quais foram organizados em 5 grupos classificatórios preconizados pela CETESB/ P4.262 ${ }^{(3)}$, como produtos geradores de resíduos químicos perigosos.

Os resultados indicam a predominância de princípios ativos no grupo dos demais produtos perigosos que representam os produtos geradores de resíduos químicos perigosos com uma ou mais características de periculosidade como inflamabilidade, corrosividade, reatividade ou toxicidade.

Tabela 3 - Frequência dos princípios ativos de produtos químicos perigosos, segundo os Grupos da Norma CETESB/ P4.262. São Paulo, 2008

\begin{tabular}{|c|c|c|c|}
\hline Grupo & Princípio Ativo & $\mathrm{n}$ & $\%$ \\
\hline Desinfetantes & $\begin{array}{l}\text { Ácido peracético, Álcool } \\
\text { etílico }\end{array}$ & 2 & 10,52 \\
\hline $\begin{array}{l}\text { Efluentes de } \\
\text { processadores }\end{array}$ & Fixador, Revelador & 2 & 10,52 \\
\hline Esterilizantes & $\begin{array}{l}\text { Glutaraldeído, hipoclorito } \\
\text { de sódio }\end{array}$ & 2 & 10,52 \\
\hline $\begin{array}{l}\text { Demais } \\
\text { produtos }\end{array}$ & $\begin{array}{l}\text { Ácido acético, Ácido ní- } \\
\text { trico, Ácido tricloroa- } \\
\text { cético, Benzina, Cloro- } \\
\text { fórmio, Éter sulfúrico, } \\
\text { Acetona,Formaldeído, } \\
\text { Óxido Nítrico, Peróxido } \\
\text { de Hidrogênio, Tintura de } \\
\text { Benjoin, Vaselina, Xilol }\end{array}$ & 13 & 68,42 \\
\hline Total & & 19 & 100 \\
\hline
\end{tabular}

Cogitare Enferm. 2013 Jan/Mar; 18(1):109-16 


\section{DISCUSSÃO}

Os produtos químicos da tabela 1 são classificados como pertencentes ao Grupo B ${ }^{(2)}$, pois são os produtos geradores de resíduos, contendo substâncias químicas que podem apresentar risco à saúde do trabalhador, saúde pública e meio ambiente, em função do potencial de inflamabilidade, corrosividade, reatividade e toxicidade. A toxicidade dos princípios ativos nos diversos órgãos e sistemas do corpo humano envolve o potencial teratogênico, carcinogênico, oftalmotóxico, neurotóxico, hepatotóxico, nefrotóxico, gastrotóxico, imunotóxico, ototóxico, cardiotoxicidade e toxicidade hematopoiética, vascular, neuropsíquica e dermo-respiratória ${ }^{(2-4)}$.

A caracterização dos produtos geradores de RQP ainda gera polêmica no contexto nacional, em função dos critérios estabelecidos pelos dispositivos legais da ANVISA, CETESB, CONAMA, CVS e NR32.

Polêmica essa evidenciada na tabela 1 com o total de 134 princípios ativos triados como produtos químicos perigosos, segundo o Grupo B da RDC 306/04(2), em comparação com a tabela 2 que traz como produtos medicamentosos geradores de resíduos perigosos, somente 13 princípios do Grupo B sendo os mesmos distribuidos entre os hormonais, antibióticos, antirretrovirais, antineoplásios e grupo dos demais produtos perigosos, segundo a $\mathrm{CVS} / 21^{(4)}$.

Fato esse preocupante uma vez que observa-se na tabela 1, por meio dos critérios da RDC 306/04 da ANVISA $^{(2)}$, outros grupos de princípios ativos que deveriam ser comtemplados pela CVS $/ 08^{(4)}$ como imunomoduladores, imunossupressores, antirretrovirais, digitálicos e medicamentos controlados, além dos hormonais, antibióticos, antir-retrovirais e antineoplásios, criteriados.

Observou-se na tabela 3, por meio do dispositivo legal da CETESB/Norma P4.262(3), um total de 19 princípios ativos geradores de resíduos perigosos, envolvendo os grupos efluentes de processadores de imagem, desinfetantes, esterilizantes e demais produtos perigosos. A caracterização de 19 princípios ativos geradores de resíduos perigosos pela CETESB indica a harmonização com os critérios da RDC 306/04 ${ }^{(2)}$ da ANVISA, uma vez que a norma da CETESB é voltada para princípios ativos perigosos não medicamentosos sistêmicos. Para os dispositivos legais CONAMA $^{(7)}$ e NR32 ${ }^{(10)}$ os 134 princípios ativos triados na tabela 1 , destacam-se como geradores de resíduos perigosos, uma vez que tais dispositivos são compatíveis com o estabelecido pela RDC 306/04 ${ }^{(2)}$ da ANVISA, fato que confirma uma necessidade de harmonização de critérios na caracterização dos produtos geradores de resíduos químicos perigosos com CVS 21/ $08^{(4)}$

Identifica-se assim que a maior dificuldade envolve a caracterização, ou não, de um medicamento sistêmico como potencial gerador de RQP. Sendo assim, cabe uma análise detalhada sobre o importante dispositivo legal para caracterização dos medicamentos geradores de RQP, representado pela Portaria CVS 21/08(4), que contempla um total de 147 produtos, desses 95 classificam-se como antineoplásicos.

A Portaria CVS 21/08 ${ }^{(4)}$ foi desenvolvida pela Divisão de Ações sobre o Meio Ambiente, o Centro de Vigilância Sanitária, com a colaboração das Divisões de Vigilância Sanitária do Trabalho, Serviços de Saúde e de Produtos e do Núcleo de Toxicovigilância. Sendo elaborada em função da observação quanto à lacuna existente na regulamentação nacional e estadual sobre a identificação dos medicamentos, cujos resíduos constituem risco significativo para à saúde dos trabalhadores, saúde pública e meio ambiente. Os objetivos da Portaria envolvem: Classificar os RSS derivados do uso de drogas ou medicamentos, criando o subgrupo Resíduos Perigosos de Medicamentos (RPM), como parte do Grupo B dos Resíduos Químicos de Serviços de Saúde; Estabelecer requisitos mínimos aplicáveis ao manejo dos RPM, para prevenir danos à saúde dos trabalhadores, à saúde pública e ao meio ambiente; Promover o gerenciamento seguro dos RPM, desde a segregação até a destinação final; Orientar quanto ao cumprimento dos regulamentos federais e estaduais sobre os RSS; e Subsidiar o PGRSS.

Os RPM são classificados como químicos que apresentam risco à saúde ou ao meio ambiente. São exemplos de RPM, entre outros: agulhas, seringas e demais dispositivos para punção venosa, equipos e conjuntos de infusão, ampolas e frascos, algodão, frascos de soro e soluções, esparadrapos e adesivos, cateteres,em geral, filtros High Efficiency Particulate Air (HEPA), materiais de limpeza e de contenção de derramamentos e acidentes, máscaras, luvas, quando contaminadas ou que tiveram contato com medicamentos listados na CVS 21/08 ${ }^{(4)}$, como Princípios Ativos que conferem periculosidade aos RSS. São também considerados RPM os medicamentos criteriados pela CVS 21/08 $8^{(4)}$, quando vencidos, ou parcialmente utilizados, e excretas de pacientes (fezes e urina), tratados com esses medicamentos, quando não passíveis de destinação por sistema de esgotamento sanitário.

Os resíduos de produtos farmacêuticos, medicamentos e similares, que não atendam aos critérios descritos da CVS 21/08 ${ }^{(4)}$, não são RPM, conforme definição ado-

Cogitare Enferm. 2013 Jan/Mar; 18(1):109-16 
tada na referida norma técnica. A Norma CVS-21/08(4) classifica ainda os RPM em dois tipos: Tipo 1 e Tipo 2, fundamentada pela NBR $10.004 / 04^{(6)}$ e pelo National Institute of Occupational Safety and Health (NIOSH), o qual estabelece que cabe a cada instituição elaborar sua própria lista de produtos perigosos ${ }^{(11)}$.

Considera-se ainda que há necessidade urgente de revisão dos critérios da Norma CVS $21 / 08^{(4)}$, no sentido de também atender à RDC 306/04 (2), no tocante à classificação desses resíduos. Destaca-se, também, que a referida Resolução, por ser mais restritiva, deva prevalecer no Gerenciamento dos Resíduos Químicos Perigosos. Assim, CVS 21/08 ${ }^{(4)}$ representa um importante dispositivo legal a ser cumprido pelas instituições de forma complementar no gerenciamento dos RQP.

Relativo à caracterização dos RQP por meio da Norma P4.262 ${ }^{(3)}$, que dispõe sobre o gerenciamento de resíduos químicos provenientes de estabelecimentos de serviços de saúde, destaca-se o fato da mesma não ser aplicável a radioterápicos, resíduos farmacêuticos, drogas quimioterápicas e materiais contaminados pelos mesmos. Ao aplicar a referida norma, cada estabelecimento deverá identificar as substâncias que efetivamente manuseia, visando a avaliação dos riscos nos resíduos gerados. Recomenda, ainda, a consulta aos fabricantes dos insumos utilizados, de modo a obter as informações necessárias para a classificação e o gerenciamento dos resíduos, avaliando suas propriedades de inflamabilidade, corrosividade, reatividade e toxicidade.

Apoiada no princípio da precaução, a Norma P4.262 ${ }^{(3)}$ estabelece que o gerador poderá optar por classificar seu resíduo como perigoso, desde que devidamente justificado. Neste caso, será dispensada a realização de ensaios para a classificação do resíduo, devendo, no entanto, o gerador informar, na identificação a ser colocada no recipiente, os seguintes dados: composição aproximada, caracterização dos resíduos quanto à presença de poluentes ambientais e característica de periculosidade.

A destinação dos RQP depende da aprovação da CETESB. Dentre as formas de tratamento e destinação de resíduos químicos perigosos, citam-se: tratamento externo para recuperação, tratamento para descarte, incineração e aterro industrial. Deve ser realizada em sistemas (tratamento, disposição ou ambos) licenciados pela CETESB.

A NR 32/05(10), importante dispositivo legal com diretrizes para o gerenciamento dos RQP, estabelece que no Programa de Prevenção de Risco Ambiental dos Serviços de Saúde deve constar inventário carac- terizando os RQP que indiquem risco à segurança e à saúde do trabalhador. A referida norma estabelece que seja elaborada uma ficha descritiva dos RQP, contendo informações tais como: características e forma de utilização do produto; riscos à segurança, à saúde do trabalhador e ao meio ambiente; medidas de proteção coletiva, individual e controle médico da saúde dos trabalhadores; condições e local de estocagem; procedimentos em situações de emergência; disponibilização de uma cópia da ficha nos locais onde o produto é utilizado. Ainda segundo a NR 32/05 ${ }^{(10)}$, o Programa de Controle Médico de Saúde Ocupacional precisará considerar as informações contidas nas fichas de RQP e o empregador deverá capacitar os trabalhadores envolvidos no manejo dos resíduos.

Evidenciou-se no presente estudo que a RDC 306/04 da ANVISA ${ }^{(2)}$, Resolução CONAMA 358/05(7) a CETESB/P4.262 ${ }^{(3)}$ e a NR32/05(10) encontram-se harmonizadas. Já a Resolução CVS $21 / 08^{(4)}$ caracteriza parcialmente os princípios ativos de medicamentos sistêmicos geradores de resíduos perigosos. É importante apontar que os diferentes dispositivos legais buscam o consenso quanto à classificação dos RQP.

A caracterização correta dos RQP indica o comprometimento do Brasil em relação ao atendimento ao Protocolo de Kyoto, no tocante ao gerenciamento dos RSS. O protocolo de Kyoto trata do comprometimento de uma série de nações industrializadas a reduzir suas emissões em 5,2\%, comparadas aos níveis de 1990, para o período de 2008 a $2012^{(12)}$.

Estudos apontam a polêmica existente, quanto aos critérios de classificação, para estabelecer a periculosidade dos RSS, em especial, os RQP no contexto nacional. Tal polêmica indica que não há um consenso na classificação dos Resíduos Químicos, sendo a problemática diretamente proporcional ao desenvolvimento de novas tecnologias, que implicam a produção, cada vez maior de RQP, produzidos em função do desenvolvimento da indústria química e farmacêutica, tanto nacional como estrangeira ${ }^{(13)}$.

Os resíduos provenientes de produtos vencidos e reagentes diversos representam uma problemática que pode tornar-se ainda mais grave, quando for medicamento caracterizado como perigoso, portanto, com potencial para gerar $\mathrm{RQP}^{(14)}$.

No Estado de São Paulo, as orientações prescritas pela CVS $21 / 08^{(4)}$, endossando as disposições da ABNT no que tange ao gerenciamento dos RSS, desde sua geração até a apresentação à coleta municipal, envolvem uma sequência de passos, iniciando-se pela classificação. 
Esta se baseia nas características e no potencial de risco dos resíduos produzidos pelo estabelecimento gerador, com o objetivo de conferir segurança em seu manuseio ${ }^{(1)}$.

Desde a década de 1980, as tentativas de estabelecer formas de gerenciamento dos RSS foram trabalhadas no sentido de criar critérios de classificação, fato este de grande relevância ao tratar da caracterização dos RQP. Durante um curto período de anos, muitas resoluções sobre os RSS surgiram e, com elas, as dúvidas inclusive sobre os produtos químicos perigosos o que tem levado os profissionais de várias categorias a buscarem soluções para problemas comuns nas instituições, como a falta de estrutura física, escassez nos recursos financeiros e preocupação com o meio ambiente ${ }^{(1,15)}$.

Os vários dispositivos legais existentes para classificar e identificar os RQP impactam diretamente em seu manejo, uma vez que este depende da segregação correta dos resíduos para a promoção da saúde humana e ambiental. Tanto no Brasil, como no exterior, o problema do gerenciamento dos RSS é polêmico, pois, além de diversos tipos de classificação, existem várias agências e serviços, de esfera federal e estadual, com legislações e regulamentações não convergentes, o que torna ainda mais difícil a organização de sistemas de administração específicos ${ }^{(16)}$.

A classificação dos resíduos é uma atividade complexa, representa um desafio, inclusive nos países desenvolvidos. Assim devem-se estabelecer critérios rigorosos para caracterizar o perigo dos resíduos de saúde ${ }^{(17)}$. Sendo os RSS de natureza heterogênea, faz-se necessária a classificação para sua segregação, possibilitando o planejamento do manejo adequado e executado com sucesso pelos hospitais ${ }^{(18)}$. Esses resíduos são gerados em todas as áreas hospitalares sendo submetidos a critérios específicos de classificação e tratamento, tanto no Brasil quanto no exterior ${ }^{(16)}$. Baseado nas características e no volume dos resíduos, o PGRSS deve ser elaborado estabelecendo as diretrizes de manejo para os mesmos $^{(19)}$. A interação do trabalhador de enfermagem com instrumentos de trabalho no manuseio de RQP indica a exposição e os riscos de doenças no trabalho ${ }^{(20)}$.

Relativo aos resíduos do grupo B percebe-se a adoção de condutas diferenciadas e que também não atendem a legislação vigente, o que indica que esta deficiência tem seu início na identificação que envolve a classificação dos $\mathrm{RQP}^{21}$. Depois de adotada a caracterização dos RSS, o próximo passo para elaborar o PGRSS refere-se a segregação que significa separação entre os diferentes grupos, e que deve ser efetuada na fonte geradora no momento de sua geração pelo próprio funcionário que realizou o procedimento ${ }^{(14)}$.
Os dispositivos legais estabelecem como RQP os derivados de produtos com potencial carcinogênico, mutagênico, teratogênico assim como os com propriedades inflamáveis, corrosivas e reativas. No entanto, a legislação brasileira, além de conflitante, ainda é insuficiente em sua aplicação(21-22). Tal situação requer ações administrativas para diminuir o impacto na saúde dos trabalhadores e saúde pública ${ }^{(23-25)}$.

O presente estudo confirma que a caracterização dos produtos geradores de RQP provoca dúvidas e impossibilita a adoção de práticas eficazes para o gerenciamento intra e extra estabelecimento de saúde,. Isso se deve à falta de critérios claros a serem estabelecidos pelos dispositivos legais no tocante a identificação de periculosidade dos produtos, com destaque os medicamentos geradores de resíduos perigosos.

\section{CONCLUSÃo}

O estudo nos permite fazer algumas considerações quanto a caracterização dos produtos de uso médico-hospitalar, contendo substâncias com potencial para geração de RQP. Identificou-se nos estoques dos almoxarifados e farmácia aqueles que caracterizam periculosidade à saúde humana e ao meio ambiente, por serem do Grupo B. Na análise de importantes dispositivos legais criados para atender a caracterização dos produtos geradores de RQP, observou-se que a RDC306/04, por ser mais restritiva, deva ser o norteador na caracterização de periculosidade sendo, no entanto observado a necessidade de atendimento à CETESB e à CVS.

Concluiu-se que os produtos químicos identificados representaram o universo de produtos geradores de RQP existentes no ambiente hospitalar a serem submetidos ao PGRSS da instituição. Verificou-se que torna-se urgente a necessidade de harmonização entre os critérios da ANVISA na caracterização dos produtos geradores de resíduos químicos e os demais dispositivos legais. Estudos já realizados sobre a saúde do trabalhador de saúde apontam para existência de polêmicas e controvérsias na caracterização de periculosidade dos produtos geradores de Resíduos de Serviços de Saúde e dentro desse universo os RQP.

\section{REFERÊNCIAS}

1. Takayanagui AMM. Trabalhadores de saúde e meio ambiente: ação educativa do enfermeiro na conscientização para gerenciamento de resíduos sólidos [tese]. Ribeirão Preto (SP): Universidade de São Paulo; 1993. 
2. Agência Nacional de Vigilância Sanitária (ANVISA). RDC n.306, de 07 de dezembro de 2004. Dispõe sobre o regulamento técnico para o gerenciamento de resíduos de serviços de saúde [Internet]. Brasília; 2004. [acesso em 26 set 2010]. Disponível: http://www.anvisa.gov.br/eng/index.htm.

3. Tecnologia de Saneamento Ambiental (CETESB). n. P4.262, de agosto de 2007. Dispõe sobre o gerenciamento de resíduos químicos provenientes de estabelecimentos de serviços de saúde. In: Companhia de Tecnologia de Saneamento Ambiental (CETESB, SP). São Paulo;2007. p. 1-13

4. Centro de Vigilância Sanitária (CVS). n. 21, de 10 de setembro de 2008. Dispõe sobre a norma técnica sobre gerenciamento de resíduos de medicamentos em serviços de saúde. In: Centro de Vigilância Sanitária (CVS, SP) São Paulo; 2008. p. 1-51.

5. Associação Brasileira de Normas Técnicas (ABNT). NBR 14725-4 Produtos químicos - Informações sobre segurança, saúde e meio ambiente. Parte 4: Ficha de informações de segurança de produtos químicos FISPQ. Rio de Janeiro: 2009.

6. Associação Brasileira de Normas Técnicas (ABNT). NBR 10.004. Resíduos sólidos - classificação. Rio de Janeiro; 2004.

7. Brasil. Conselho Nacional do Meio Ambiente (CONAMA). Resolução n.358, de 29 de abril de 2005. Dispõe sobre o tratamento e destinação final dos resíduos dos serviços de saúde. In Conselho Nacional de Meio Ambiente (CONAMA, BR). Brasília; 2005. p.1-8.

8. Agência Nacional de Vigilância Sanitária - ANVISA. Portaria n.344, de 12 de maio de 1998. Dispõe sobre o Regulamento Técnico sobre substâncias e medicamentos sujeitos a controle especial. In: Agência Nacional de Vigilância Sanitária (ANVISA, BR). Brasília; 1998. p.1 - 64 .

9. Agência Nacional de Vigilância Sanitária. Manual de Gerenciamento de Resíduos de Serviços de Saúde/ Ministério da Saúde, Agência Nacional de Vigilância Sanitária. Brasília: Ministério da Saúde, 2006.

10. MinistériodoTrabalhoeEmprego(BR).NormaRegulamentadora NR 32, 11 de novembro de 2005. Dispõe sobre a segurança e saúde no trabalho em serviços de saúde. Brasília; 2005.

11. National Institute of Occupational Safety \& Health. Preventing occupational exposures to antineoplastic and other hazardous drugs in health care settings [Internet]. USA; 2004. [acesso em 29 jan 2009]. Disponível: http:// www.cdc.gov/niosh/docs/2004-165/
12. Philippi Jr A. Saneamento, saúde e ambiente: fundamentos para um desenvolvimento sustentável. Barueri: Manole, 2005.

13. Silva CE, Hoppe AE. Diagnóstico dos resíduos de serviços de saúde no interior do Rio Grande do Sul. Eng. Sanit. Ambient. 2005;10(2):146-51

14. Costa AMP. Elaboração e avaliação da implantação de um "Modelo Básico" de plano de gerenciamento de resíduos de serviços de saúde em unidades hospitalares da Região Metropolitana da Baixada Santista. [dissertação]. São Paulo (SP): Universidade de São Paulo; 2001.

15. Felli VEA. Plano de gerenciamento de resíduos químicos hospitalares. Anais eletrônicos. [CD-ROM]. In: Anais do $56^{\circ}$ Congresso Brasileiro de Enfermagem. Gramado (RS) [Internet] 2004 [acesso em 29 mar 2005]. Disponível: http://www.abennacional.org.br/ eventos_aben_realizados.html

16. Takayanagui AMM. Risco ambiental e o gerenciamento de resíduos nos espaços de um serviço no Canadá: um estudo de caso. [tese]. Ribeirão Preto (SP): Universidade de São Paulo; 2004.

17. Rodrigues GAS. A enfermagem gerenciando o serviço de lixo hospitalar. Nursing rev. tec. Enfermagem. 2000;28(9):5-7.

18. Garcia LP, Zanetti-Ramos BG. Gerenciamento dos resíduos de serviços de saúde: uma questão de biossegurança. Cad. Saúde Pública. 2004;20(3):744-52.

19. Salles CLS. Acidentes de trabalho ocorridos com os trabalhadores de saúde nos diferentes processos de um plano de gerenciamento de resíduos de serviço de saúde. Cienc. Cuid. Saude. 2008;8(4):652-9.

20. Sarquis LMM, Felli VEA. Acidentes de trabalho com instrumentos perfurocortantes entre os trabalhadores de enfermagem. Rev Esc Enferm USP. 2002;36(3):222-30.

21. Costa TF, Felli VEA. Periculosidade dos produtos e resíduos químicos da atenção hospitalar. Cogitare enferm. 2012;17(2):322-30.

22. Alberguini LBA, Silva LC, Rezende MOO. Tratamento de resíduos químicos: guia prático para a solução dos resíduos químicos. São Carlos: Rima; 2005.

23. Baptista PCP, Vitorino DC, Silva FJ, Costa TF, Tito RS. Identificação de cargas de trabalho e processos de desgastes nos trabalhadores de enfermagem em São Paulo. In: XI Conferência Ibero Americana de Educação 
em Enfermagem e III Encontro Latinoamerica-Europa - ALADEFE. Coimbra; 2011. p.556.

24. Safiano CM, Sarquis LMM, Felli VEA, Giacomozzi LM. O processo saúde-doença vivenciado pelos trabalhadores de enfermagem em uma instituição hospitalar. Cogitare enferm. 2003;8(2):87-91.

35. Costa TF, Felli VEA, Baptista PCP. A percepção dos trabalhadores de enfermagem sobre o manejo dos resíduos químicos perigosos. Rev Esc Enferm USP. 2012;46(6)1453-61. 